Publié avec le concours du

Centre National de la Recherche Scientifique 



\begin{tabular}{|l|l|l|l|}
\hline DHA & 16,1 & 1990 & $259-280$ \\
\hline
\end{tabular}

\title{
CADASTRES ET ESPACE RURAL DANS LE NORD-OUEST DU PÉLOPONNÈSE *
}

\author{
A. RIZAKIS, KEPA/EIE, Athènes
}

C'est E. Kornemann ${ }^{1}$ qui a le premier considéré l'année 44 av. J.-C. comme terminus post quem de la fondation de la colonie romaine de Dymé, l'attribuant ainsi, soit à César lui-même, soit aux Triumvirs. Cette datation a été acceptée par tous les spécialistes 2 ; toutefois, ccux-ci sont en désaccord sur un point important de

* La rédaction de cet article a été facilitée par les discussions que j'ai eues, à des périodes différentes, avec G. CHOUQUER, M. CLAVELLEVEEQUE et P. DOUKELLIS: les problèmes posés par certaines émissions dyméennes, ont été discutés avec J. TOURATSOGLOU, T. HACKENS et K. LIAMBI. Les dessins sont dûs à S. ZOUMBAKI et la correction du texte à Y. RIZAKIS. A toutes et à tous j'exprime, ici, mes profonds remerciements.

1. RE IV 1, 1900, col. $530 \mathrm{n}^{\circ} 10$.

2. Cf. F. BÖLTE, RE, V. 2 et E. MEYER Kleine Pauly II, s.v. Dyme; M. GRANT, From Imperium to Auctoritas, Cambridge 1946 (réimpression 1969), 264: Fr. VITTINCHOFF, Römische Kolonisation und Bürgerrechtspolitik unter Caesar und Augustus, Wiesbaden 1952, 86 ; M. AMANDRY, Le monnayage de Dymé (Colonia Dumaeorum) en Achaïe. Corpus (Pl.XIII-XVI), RN 23, 1981,55 . 
l'histoire coloniale de Dymé; en dehors de la refondation par Auguste, universellement admise, la cité en aurait connue, d'après M. Grant, une seconde sous Antoine qui serait attestée, d'après lui, par une émission rare dont une seule pièce est, actuellement, conservée au Musée des Médailles 3 . Cette hypothèse, qui a provoqué la méfiance voire la vive protestation des historiens de la colonisation romaine 4 , a été reprise tout récemment par M. Amandry dans son étude exemplaire du monnayage de la colonie ${ }^{5}$. Malgré les efforts et les progrès réalisés dans ces domaines par les études récentes, les réponses ne sont pas définitives; notre propos n'est pas de résoudre le problème mais d'ajouter quelques indices complémentaires qui pourraient éclairer certains aspects de la question et donner une autre orientation dans la réflexion générale sur le problème de la colonisation et des refondations.

Les numismates datent une double émission coloniale de Dymé, qui est sans doute la plus ancienne connue de la cité, vers l'année 40 av. J.-C. Etant donné que les monétaires qui y figurent ont la fonction des IIviri Quinquennales, ces spécialistes montent, à très juste titre, la date de la fondation vers l'année 44 av. J.-C. 6 . Certains spécialistes vont même plus loin en mettant en rapport la fondation de Dymé avec celle de Corinthe, c'est-à-dire, en considérant les deux fondations comme contemporaines et comme l'oeuvre de César lui-même 7 . Cette dernière hypothèse est très

3. M. GRANT, o.l., 264-265; et aussi M. AMANDRY RN 23, 1981, 45-67 et particulièrement p. 51 (III) et 57-58 ; cf. aussi ID., Monnayage émis en Achaïe sous l'autorité d'Antoine (40-31), Proceedings of the International Numismatic Convention on Greek Imperials, Israel Numismatic Journal 6-7, 1982/3, 2.

4. Cf. Fr. VITTINGHOFF, C.R. sur la monographie de M. GRANT (p. 259 n.2.), Gnomon 1950, 264 et ID., (cit. n. 2) 86, n. 4. Cf. aussi cidessous p. 264 note 23.

5. Pour le monnayage de Dymé voir la notice complémentaire du même auteur. Une nouvelle émission dyméenne (PI.X), RN 25, 1983, 53-56. Nous avons nous-même adopté la thèse de $M$. GRANT [cf. A. RIZAKIS, Études sur l'Achaie romaine II (Lyon 1979, thèse inédite), 240] mais le réexamen des documents nous oblige à prendre, maintenant, des distances.

6. Pour la description de ces pièces et la justification de leurs dates voir M. AMANDRY, RN 21, 1981, 50-51 (I et II) et 52-57.

7. M. AMANDRY, $R N, 23,1981,55$. Pour la fondation de Corinthe et son monnayage voir maintenant M. AMANDRY, Le monnayage des 
vraisemblable et est plus ou moins confirmée par une source littéraire qui avait échappé jusqu'à présent à l'attention des savants ; il s'agit d'une petite notice, noyée dans une lettre que Cicéron adressa le 8 juillet 44 av. J.-C. à son ami Atticus; le ton général de la lettre exprime la déception et l'amertume de l'orateur voire son désespoir pour l'évolution des affaires politiques de Rome; il y critique très sévèrement les meneurs du jeu politique et leurs décisions. La notice en question exprime la crainte de l'orateur que les pirates de Dymé, installés dans cette cité par Pompée en 67 av. J.-C., ne reprennent de nouveau la mer après avoir été expulsés par les colons romains : Dymaeos agro pulsos mare infestum habere nil mirum 8 ; l'installation des colons romains était déjà réalisée quand Cicéron écrit sa lettre à Atticus mais on ne saurait affirmer si la procédure de la deductio avait été complétée avant ou peu après Mars 44 av. J.-C. ; il ne semble pas que la réaction des Dyméens, à cette entreprise d'expropriation de leurs terres, fut violente comme dans le cas contemporain de Buthrote, en Epire, où les colons furent chassés par les habitants révoltés 9; Cicéron présente, dans sa lettre, les pirates de Dymé ainsi que les petits propriétaires de Buthrote comme les victimes de la politique coloniale insensée de César et de ses lieutenants 10 . L'opposition entre Pompée le grand et César est flagrante: la générosité du premier a permis aux pirates de retrouver dans le travail de la terre une vie pacifique sans danger;

duovirs corinthiens (BCH, Supplément 15), Athènes-Paris 1988 passim.

8. Cic., Ad Att., XVI, 1.

9. Cic. Ad Att., XV. 29,3 ; XVI.1 et 4 ; la colonisation de Buthrote n'a pas été réalisée par le dictateur; l'intervention de Cicéron en faveur de ses amis de Buthrote a empêché dans un premier temps l'envoi des colons; Cf. E. DENIAUX, Un exemple d'intervention politique : Cicéron et le dossier de Buthrote en 44 av. J.-C., Bull. Ass. G. Budé 2, 1975, 283-296; EAD. Atticus et l'Epire in L'Illyrie méridionale et l'Epire dans l'Antiquité, Actes du colloque international de Clermont-Ferrand (22-25 Octobre 1984), Clermont-Ferrand, Adosa 1987, 245-249; la deductio d'une colonie romaine a été réalisée deux ans plus tard; cf. VITTINGHOFF (cit.p. 259 n.2), 86 ; P.A. BRUNT, Italian Manpower, 225B.C.-A.D.14, Oxford 1987, 598.

10. Sur la politique de la colonisation césarienne des côtes occidentales grecques voir A. RIZAKIS, A contribution to the History of Roman Colonization in the N.W. Peloponnese (en grec avec résumé en anglais dans ПOIKIAA, MELETIMATA 10, à paraître). 
leur expulsion violente des terres de Dymé qu'ils ont mises en valeur, ne risquerait-elle pas de les rejeter dans les mers afin de reprendre leur métier d'antan 11 ?

Le problème des refondations coloniales à Dymé, évoqué seulement par quelques émissions monétaires, est plus compliqué. Parmi ces émissions, seules celles qui datent de la période augustéenne ne posent aucun problème de datation ou d'attribution à Dymé car elles portent le portrait du vainqueur d'Actium avec la nouvelle dénomination de la colonie: C(olonia) J(ulia) A(ugusta) Dum(aeorum) 12. Cette nouvelle deductio s'inscrit parfaitement dans la nouvelle politique d'Octave après sa victoire contre Antoine; les exemples semblables sont nombreux en Grèce et en Orient en général 13 . En revanche la deductio, attribuée à Antoine, est contestable et les objections ne sont pas seulement d'ordre politique ou juridique mais, comme on va le voir, numismatique. En fait cette hypothèse est appuyée sur une seule émission dyméenne qui porte, au droit, le buste casqué de Pallas-Athéna et, au revers, la légende C.I.A. DUM 14. L'attribution de cette émission à la période antonienne est fragile, malgré les éléments que le dernier auteur évoquait en faveur de cette thèse, à savoir, en dehors de quelques détails techniques 15 , l'identité du type du droit avec l'émission II (qui date des années 40 av. J.-C.) et l'absence à la fois du portrait d'Auguste (ou du moins du noms du Princeps) et des noms des IIviri Quinquennales qui figurent sur les premières émissions coloniales mais sont absentes, il est vrai, des émissions augustéennes 16. En fait, aucune de ces considérations ne peut être décisive. Certes, nous avons,

11. Pompée avait installé à Dymé, en 67 av. J.-C., une partie des pirates vaincus; les sources (Strab. XIV, C 665 ; Plut., Pomp. 28,7) ne précisent pas le caractère de cette installation: s'agit-il d'une colonie?

12. CF. M. AMANDRY, RN, $21,1981,52$ (V) et 58-59.

13. Cf. Fr. VITTINGHOFF (cit. p. 259 n.2), 126-129. Il semble que dans cette phase Octave n'a pratiquement pas créé de nouveaux établissements; il procéda à des refondations dans les anciennes colonies de César ou des triumvirs; chaque restitutio était accompagnée par l'installation de nouveaux colons (cf. GRANT, cit. p. 259 n. 2, 305).

14. Cf. AMANDRY, RN 21, 1981, 51(III) et 57-58.

15. Cette dénomination qui est un semis est semblable d'après AMANDRY (o.l., 58) aux "semisses de l'émission corinthienne frappée par P. Aebutius et C. Pinnius entre 39 et 36 avant J.-C.".

16. Cf. AMANDRY, RN 21, 1981, 57. 
au droit des deux émissions, le même type iconographique, mais la ressemblance stylistique des bustes casqués d'Athéna n'est pas très convaincante (cf. Amandry pl. XIV) 17. Cette différence s'expliquerait mieux, par exemple, s'il s'agissait d'une pièce anniversaire frappée ultérieurement, pendant la période augustéenne ; de telles émissions sont connues sous l'Empire soit pour les émissions de Rome soit pour celles des cités de l'Orient 18 . Une telle hypothèse justifierait, en dehors des absences signalées cidessus, la présence d'autres éléments qui se rattachent probablement à la période augustéenne. Il s'agit en premier lieu, de la forme de l'abréviation du nom complet de la colonie [C.I.A.DUM] qui ne figure que sur les émissions augustéennes; cette forme, plus claire que la précédente [C.I.D.], surtout en ce qui concerne l'ethnique, est absolument nécessaire après Actium, lorsque la confusion avec les colonies de Dyrrachium et de Dium, en Macédoine, était possible 19. Cela expliquerait aussi l'utilisation d'une autre forme abrégée de l'ethnique DIMEN(sis) sur une émission dyméenne qui date des années 29 ou 28 av. J.-C. et qui associe Octavien au droit avec la tête diadémée de César sur le revers 20 . Cette forme d'ethnique correspond mieux à l'usage du latin et est connue par les inscriptions contemporaines 21. Enfin, il faut signaler que cette légende est ceinte

17. Les caractéristiques de la tête casquée d'Athéna rappellent la conception stylistique des portraits de la première période augustéenne: nez pointu, bouche étroite et surtout un long et puissant cou; l'ensemble est rendu avec une régularité exemplaire.

18. Voir en général M. GRANT, Roman Anniversary Issues, New-York 1977, 18-30 ; J. TOURATSOGLOU, Die Münzstädte von Thessaloniki in der römischen Kaiserzeit, 1988, 26-29.

19. La date exacte de la première fondation de ces colonies macédoniennes n'est pas certaine; GRANT (cit. p. 259 n.2, 272-278) les attribue à la période triumvirale en les considérant comme l'ocuvre d'Hortensius et d'Antoine respectivement. Il semble qu'elles connurent une nouvelle restitutio après Actium (ci-dessous p. 266) et elles portent toutes les deux l'épithète d'Augusta. Cf. AMANDRY, $R N, 21,1981,53$ qui discute des problèmes de l'attribution des deux premières émissions dyméennes qui portent la légende C.I.D. plutôt à Dymé qu'à Dium en Macédoine.

20. Cf. AMANDRY, $R N, 25,1983,55-56$.

21. Cf. A. RIZAKIS, RPh LXX, 1985 fasc. $1,87-88 n^{\circ} 46$. 
d'une couronne qui constitue, indiscutablement, un élément numismatique caractéristique de la période augustéenne 22.

La légende, sur cette pièce, doit donc être interprétée $C$ (olonia) $J$ (ulia) A(ugusta) Dum(aeorum) et non plus C(olonia) J(ulia) A(ntonia) Dum(aeorum), comme cela a été jusqu'alors supposé ; ce qui implique son classement dans la période augustéenne. Ce classement nous évite l'inutile effort de justifier une irrégularité d'Antoine qui n'aurait aucun parallèle, du moins, en Orient ${ }^{23}$. Il serait vraiment étonnant qu'un tel acte illégal soit complètement ignoré par les sources hostiles à Antoine, qui sont par ailleurs très bavardes quand il s'agit de dénoncer d'autres actes moins graves du triumvir. Dymé se trouvait, certes, dans la zone contrôlée, avant Actium, par Antoine qui avait montré de bonne heure son intérêt pour la région et qui avait réussi à créer une solide clientèle dans beaucoup de cités d'Achaïe 24. Cependant, il ne semble pas qu'elle ait occupé une place

22. Cf. C.H.V. SUTHERLAND and C.M. KRAY, Catalogue of Coins of the Roman Empire in the Ashmolean Museum Oxford, Part. I. Augustus (c. 31 B.C-A.D.14), Oxford 1975 passim.

23. Cf. E. GABBA, Colonie triumvirali, 101sq. qui pense également que les exemples de colonies d'Antoine évoqués par GRANT (cit.p. 259 n.2., 264 et $274 \mathrm{sq}$.) sont douteux; Cf. en dernier lieu L. KEPPIE, Colonization and Veteran Settlement in Italy, 1980, $17 \mathrm{n}$. 4 : "The coin legends adduced by Grand $(264,274 \mathrm{sq}$.) are of incertain relevance". AMANDRY ( $R N$ 21, 1981, 57) évoque le cas de Casilinum, colonie de César, où Antoine procéda à une nouvelle deductio malgré les conseils contraires de Cicéron (Phil. II, 102), mais cet argument ne saurait être décisif.

24. Antoine n'a pas reconnu l'accord de Misène ( 39 av. J.-C.) aux termes duquel Octave accordait à Pompée l'Achaie qui ne faisait pas partie de ses possessions. L'installation d'une garnison dans l'île de Zante, en 39 av. J.-C., déjà était destinée à l'empêcher de prendre possession du Péloponnèse; le monnayage et les inscriptions montrent son intérêt pour plusieurs cités où il a réussi à établir une puissante clientèle; pour les émissions monétaires antoniennes dans les différentes villes de la région voir AMANDRY R.N. 23, 1981, 55-56 avec les notes et surtout, ID., Israel Numismatic Journal 6-7, 1982/3, 1-6 avec pl.I. Sur l'influence d'Antoine à Patras voir L. MORETTI, Due patroni per Patrasso, RivPhil. 108, 1980, 448-452 où MORETTI reconstitue et commente avec beaucoup de succès une inscription honorifique, élevée par la cité de Patras en l'honneur de Censorina, épouse de L. Sempronius Atratinus - qui fut legatus pro praetore de l'Achaïe entre 39 et 37 - et fille de Lucius Marcius 
très importante dans les plans stratégiques d'Antoine; la cité ne possédait pas de port (Strab. VIII, 7,5) et n'avait jamais joué un rôle quelconque dans les communications avec l'occident. En revanche, le port de Patras, situé à 24 kilomètres à l'Est, présentait tous les avantages d'une place stratégique pour le contrôle de l'entrée du golfe de Corinthe et des communications maritimes 25 ; ce n'est pas par hasard qu'Antoine choisit Patras comme lieu d'hivernage pour son armée avant la bataille finale, à Actium 26 ; Agrippa, après avoir enlevé à Antoine plusieurs points stratégiques sur les côtes occidentales, réussit à s'emparer de Patras, une des positions clés d'Antoine pour les communications et l'approvisionnement de son armée 27. Dymé n'est nullement citée; il en est de même après Actium (30 av. J.-C.), quand Octavien s'efforça de gagner la sympathie des colonies d'Antoine 28. Si Antoine avait vraiment, contre le droit et la pratique romaine, procédé à une nouvelle deductio à Dymé, cette ancienne colonie de César, Octave n'aurait pas laissé une telle irrégularité impunie ni sa propagande sans commentaire. Il faut, donc, croire qu'Antoine n'avait pas procédé à une restitutio à Dymé car, comme on vient de le voir, il n'avait aucune raison politique ou stratégique d'agir ainsi : pour renforcer son influence à Dymé il aurait suffit de procéder à une simple adscriptio de colons, comme le conseillait dans d'autres situations Cicéron (Phil., II, 102). Un tel acte expliquerait la présence, dans la cité de Dymé, de quelques Antonii 29 , appartenant à sa clientèle personnelle ou à celle de sa famille.

La deductio d'une nouvelle colonie à Dymé par Auguste est, traditionnellement, placée après la réorganisation de la province

Censorinus - gouverneur de Macédoine et de l'Achaïe en 42-40. Sur la clientèle antonienne à Dymé voir ci-dessous n.29.

25. Cf. A. RIZAKIS, Le port de Patras et les communications avec l'Italie sous la République, Cahiers d'histoire XXXIII, 1988 N³-4, 453-472.

26. Dio Cassius L. 9, 2-3. Pendant ce séjour (32/1 av. J.-C) il fait frapper une émission en l'honneur de Cléopâtre : c'est la dernière émise sous l'autorité d'Antoine en Grèce: cf. I. N. SVORONOS, 'H

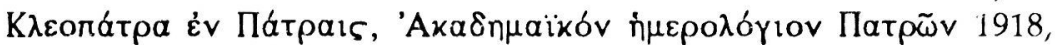
123-128; AMANDRY, Israel Numismatic Journal 6-7, 1932/3, 4.

27. Dio Cass., L. 13, 5 ; Vell. Paterc., II. 84, 2 ; cf. J.M. RODDAZ, Marcus Agrippa (BEFAR 253), 1984, 161-164.

28. Cf. L. KEPPIE, (cit., p. 264 n. 23), 114.

29. Cf. RIZAKIS (cit. p. 260 n. 5) et AMANDRY, RN 23, 1981, 58. 
d'Achaïe c'est-à-dire après 27 av. J.-C. 30, le meilleur argument de cette thèse étant les émissions augustéennes qui portent la légende révélatrice C(olonia) I(ulia) A(ugusta) Dum(aeorum) 31. Pourtant une telle datation de la restitutio augustéenne n'est pas certaine; il semble, en effet, que l'ensemble des fondations ou refondations augustéennes, dans la péninsule hellénique, aient été conçues et réalisées, pendant la période qui suivit immédiatement la victoire d'Actium, lorsqu'Octave entrepris un vaste programme de colonisation dans la région. La présence de l'épithète Augusta sur les légendes de leurs émissions ne peut pas justifier, dans certains cas du moins, l'attribution de ces fondations à une date postérieure à 27 av. J.-C. (entre 27 et 14 av. J.-C.). Dion 32 rapporte, en effet, qu'Octave installa après sa victoire (30 av. J.-C.) des partisans d'Antoine, venant d'Italie, dans d'anciennes colonies de Philippes et de Dyrrachium, mais aussi dans d'autres cités dont il ne précise pas le nom. Vittinghoff et Brun 33 ont considéré, avec raison, qu'on peut inclure dans celles-ci Cassandreia et Dium en Macédoine ; la liste pourrait être plus longue; la présence de l'épithète Augusta n'est pas en contradiction avec Dion, comme le pensait Grant ${ }^{34}$, car on peut supposer soit que celle-ci ait été donnée par le Princeps, à certaines colonies auxquelles il voulait lier ultérieurement son nom 35 , soit que, dans certains cas, le processus de la restitutio, commencé après Actium, ait duré quelques années et n'ait été complété qu'après 27 av. J.-C. 36 . Pour en revenir à Dymé une émission rare, représentée par une seule pièce du cabinet des Médailles et publiée récemment par Michel Amandry 37, associe le nouveau maître de l'Empire au culte du Divus Julius; elle porte, au droit, la

30. Cf. AMANDRY, RN 23, 1981, 59.

31. Cf. AMANDRY, o.l., 51-52 (IV) et 58-59.

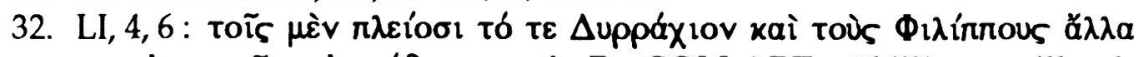

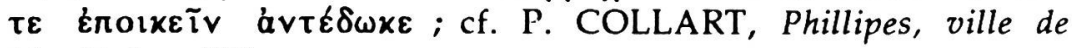
Macédoine, 229 sq.

33. VITTINGHOFF (cit. p. 259 n. 2), 126-127 ; BRUNT (cit. p. 261 n. 9), 599.

34. L'auteur considère comme erronée l'information de Dion et il arrive à la conclusion que l'ensemble des restitutiones des colonies en Macédoine et en Achaïe, qui portent l'épithète augusta datent de la période comprise entre 27 et 14 av. J.-C. (cit. p. 259 n. 2, 275, 278 et 283).

35. Cf. KEPPIE, (cit. p. 264 n. 23), 80.

36. Cf. BRUNT (cit. p. 261 n. 9), 599.

37. $R N 25,1983,55-56$. 
tête nue d'Octave et les noms des duumviri C.IULI. CALAMO/ $L$ $A E M I L L A \ldots ;$ au revers, la tête diadémée de César et la légende [C.I.] DIMEN(siis); son symbolisme est évident : "acte de pietas envers le fondateur de la colonie et reconnaissance du Princeps, le double du divin César" ; l'émission date plutôt des années 29 ou 28 sans toutefois exclure le début de l'année 27 avant qu'Octave ne prenne le titre d'Auguste 38 , mais il est vraiment dommage que cette pièce unique ne conserve pas le nom de la colonie. Vu sa datation, la restitution la plus probable de la légende qui figure sur son revers est [C.I.] DIMEN(sis) et non pas [C.I.A.] DIMEN(sis), comme on l'a suggéré ${ }^{39}$. Dymé aurait-elle reçu, à ce moment, comme certaines colonies macédoniennes, un renforcement de colons, l'épithète Augusta étant ajoutée, ultérieurement, dans sa dénomination officielle, après l'année 27 av. J.-C. 40 ?

Dymé a donc connu au Ier s. av. J.-C. trois colonisations successives : celle des pirates de Pompée en 67 av. J.-C., celle de César en 44 av. J.-C. et enfin celle du début de l'Empire; dans ce dernier cas, il est difficile d'en préciser la date. En dehors des changements politiques et sociaux, les nouvelles réalités modifient en profondeur la physionomie de l'organisation de l'espace dans cette région. L'installation des colons romains recevant des lots de terre a, nécessairement, entraîné, avec les confiscations parfois forcées, des redistributions et de nouvelles divisions du sol ; elle a conduit à la dépossession des indigènes, du moins d'une partie de leur moyen de production et d'échange en leur imposant non seulement de nouveaux cadres politiques mais aussi les instruments du contrôle politique et idéologique. Ces changements se sont concrétisés au niveau des paysages par des cadastrations successives, seuls moyens d'évaluation et de contrôle de l'espace à des fins multiples, politiques, économiques et fiscales.

$R$. Chevallier avait signalé dans une courte notice, il y a vingt ans, l'existence de centuriations romaines à Dymé; moi-même, annonçais, plus récemment 41 , le projet d'une recherche sur les interventions romaines dans le paysage agraire grec, en particulier celui des colonies romaines. La reconstitution de ces réseaux centuriés

38. AMANDRY, ibid., 56.

39. Ibid., 55.

40. AMANDRY, RN 23, 1981, 58-59, date les émissions augustéennes de Dymé des premières années du Principat, $27 / 26$ ou 25 av. J.-C.

41. R. CHEVALLIER, BCH 82, 1958, 635-636 ; A. RIZAKIS, DHA 1985, 761. 
est encore possible dans le cas dyméen ; ceux-ci sont parfois dégradés dans le paysage sous la forme d'indices topographiques à savoir : éléments de voirie et de limites parcellaires 42 . L'ampleur des traces fossiles conservées dans le paysage permet de présenter globalement les résultats provisoires de l'enquête et de proposer, avec toutes les réserves nécessaires à cette étape de recherche, une reconstitution de son extension probable.

Trois centuriations ont été repérées dans la région : nous les appellerons conventionnellement $\mathrm{A}, \mathrm{B}$ et $\mathrm{C}$. Le cadastre $\mathrm{A}$ (carte I) s'étend sur une grande partie de la plaine occidentale et sur une partie du plateau de Dymé (actuellement Kato-Achaia); au nord, il semble s'arrêter à la route qui lie actuellement Kato Achaia (anc. Dymé) avec le cap Araxos et qui correspond, comme le montre la disposition de l'habitat dans cette zone, à une voie ancienne 43 ; cette route sépare la plaine au sud, d'une région de mottes et de collines au nord en bordure de la côte. Au SO, les limites des traces coïncident avec le cours du Larissos, au NO avec la zone marécageuse et les lagunes, au SE avec les piémonts du Mt Movri et au NE avec le cours du Serdini qui constitue la limite du plateau de Dymé vers l'est. Au sein des limites que nous venons de définir nous percevons des différences de densité de signes ; celle-ci est remarquable autour des villages actuel de Limnochorion (au nord du grand axe du cadastre), Sageika, Apostoloi, Bouteika et Kareika (au sud du même axe), alors qu'il existe des étendues pauvres en signes ou vides ; fait qui demande naturellement une réponse. Le cadastre $A$

42. Pour le repérage des cadastres centuriés nous disposons de deux documents de travail en Grèce; les cartes topographiques et les photographies aériennes verticales; les premières existent à deux échelles : 1.50 .000 et 1.5000 ; les secondes sont à une échelle de 1.20.000. L'utilisation des documents cartographiques présente des inconvénients car les premières cartes ne permettent que des repérages généraux, alors que les secondes sont trop détaillées et chargées ; il est par conséquent, difficile de procéder à de vastes assemblages. Heureusement nous pouvons corriger ce défaut par une réduction des cartes à une échelle de 1.20.000.

43. Cf. M. LACAKIS-A. RIZAKIS, Polis et chôra : l'organisation de l'espace urbain et rural en Achaïe occidentale, Actes du Xe congrès international de l'archéologie classique, Berlin 24-31 juillet 1988 (sous presse) avec carte géographique et M. LACAKIS, 'A ypotiko í

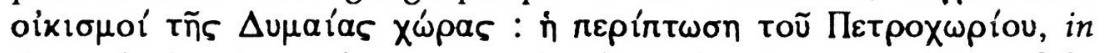
Actes $d u$ Ier Symposium International sur l'Achaie et l'Elide (19-21 Mai 1989), sous presse. 


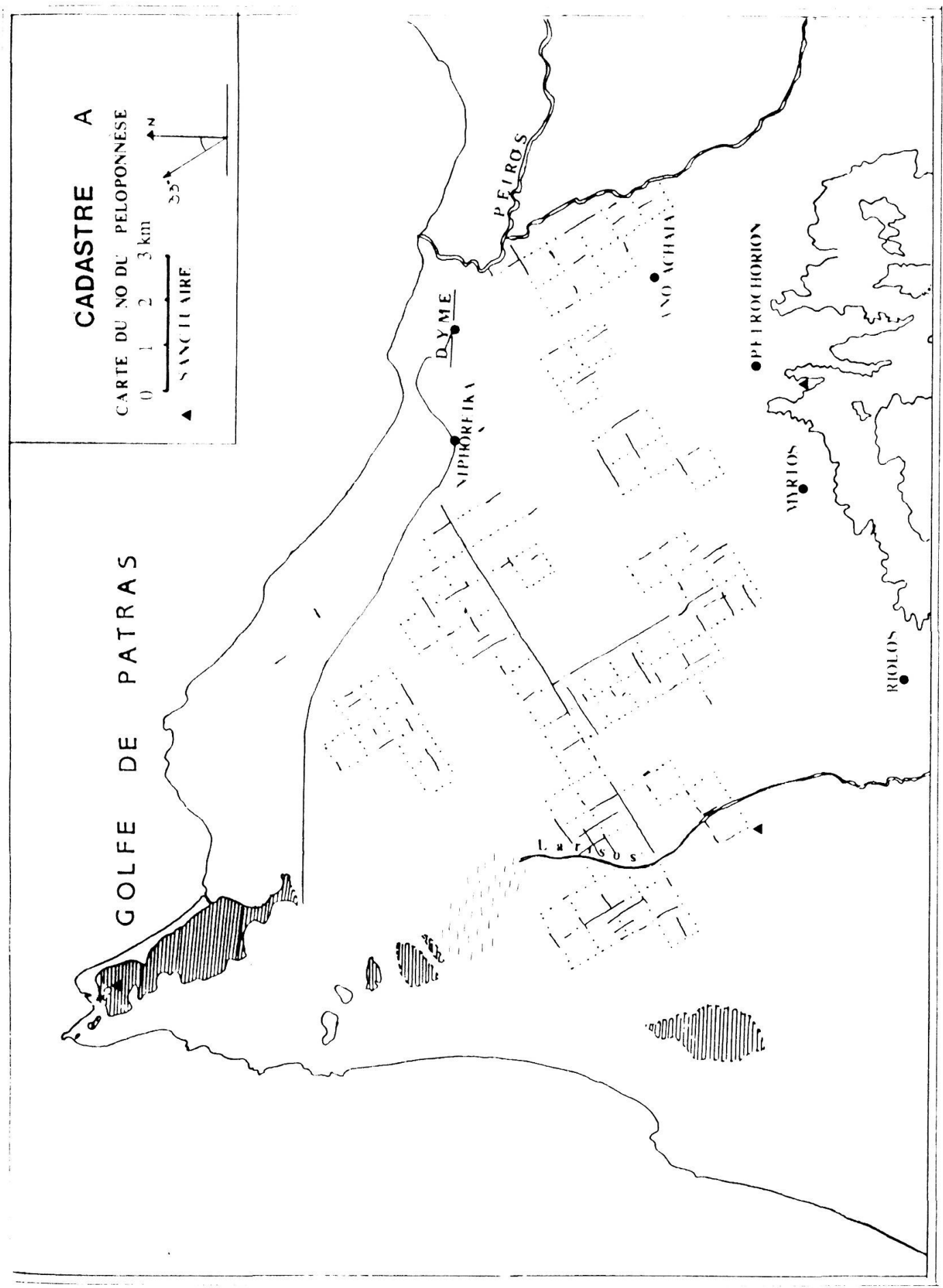


est assis sur une voie dont la photographie aérienne révèle des traces fossilisées sur le sol; elle part à proximité du village actuel de Niphoreika à l'Ouest et, après avoir traversé la plaine occidentale suivant une direction NE-SO, aboutit au village de Lappas, au delà du Larissos. En dehors de cet axe, le cadastre n'a conservé aucun autre limes parallèle de même distance alors que le paysage conserve les traces de quelques cardines sur la partie sud du territoire.

Le cadastre $B$ (carte II), qui est superposé au précédent, occupe plus ou moins la même zone c'est-à-dire, celle qui correspond à la plaine occidentale; toutefois il n'y a pas de traces de cette centuriation sur le plateau de Kato-Achaia sur la partie où s'étendait le précédent. Il est remarquable que ce cadastre soit assis, comme le précédent, sur un axe dont au moins une partie s'écarte peu de la voie actuelle du chemin de fer. Le départ de cet axe se trouve près de la sortie NO de Dymé et l'aboutissement dans la région de Lappas; il est caractéristique que ce cadastre ne conserve pas d'autres limites parallèles d'une semblable longueur mais qu'il ne présente que quelques cardines sur la partie Sud de son grand axe ; il n'est, donc, pas étonnant que la plus grande densité des signes se trouve au sud du grand axe, là où les terres sont plus fertiles.

Le cadastre C (carte III), superposé aux précédents, a une extension beaucoup plus grande car, en dehors des zones qu'occupent les deux précédents, il se déploie à l'est, vers les plateaux formés par le Peiros et ses affluents, et au SO, vers la grande plaine qui s'étend au delà du Larissos. Il est caractéristique que les traces repérées sur la plaine occidentale, occupée par les centuriations précédentes, soient éparses sinon énigmatiques; elles occupent une partie de la zone côtière vers le nord ainsi que les buttes, au SO du plateau de Dymé. La plus grande densité de signes, à l'est, se situe entre le lit du Peiros et celui de son affluent le Parapeiros, région qui appartient, certainement, au territoire de Pharai 44; des signes moins nombreux ont été repérés dans la région de Loussika dans la basse vallée du Peiros et du Serdini. La discontinuité qu'on remarque dans cette zone pourrait s'expliquer par la géomorphologie particulière de cette dernière où les effets de l'érosion sont très grands et apportent des changements constants à son relief 45 . L'orientation de cette

44. Cf. F. BÖLTE, RE XIX, 1938, col. 1796, s.v. Pharai.

45. Il est connu que le lit des fleuves a constitué souvent une zone de subseciva où, naturellement, s'interrompait le tracé des limites dont le changement du lit a contribué à dégrader le réseau, cf. E. CASTAGNOLI, La centurizzione di Lucca, SE, XX, 285 sq. 


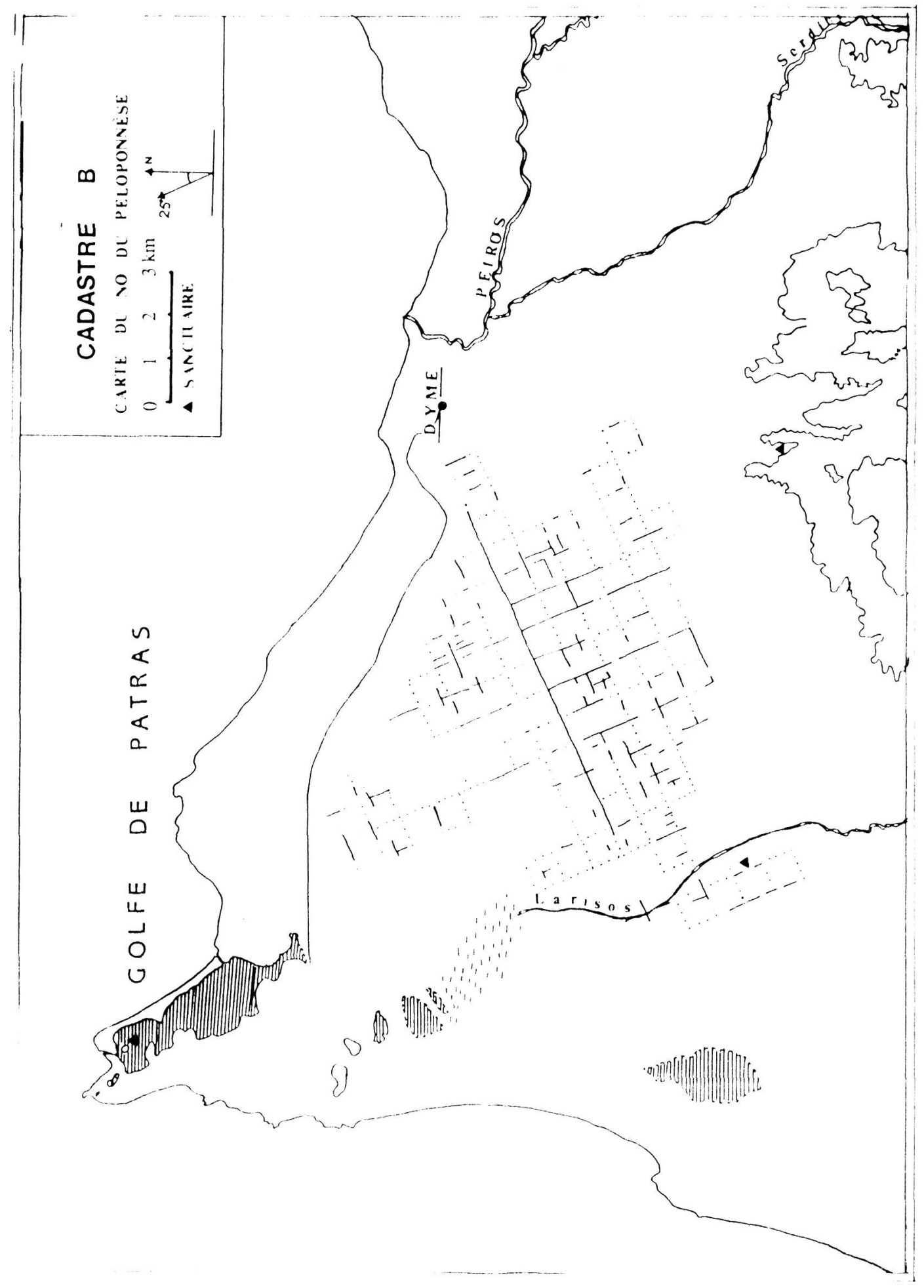




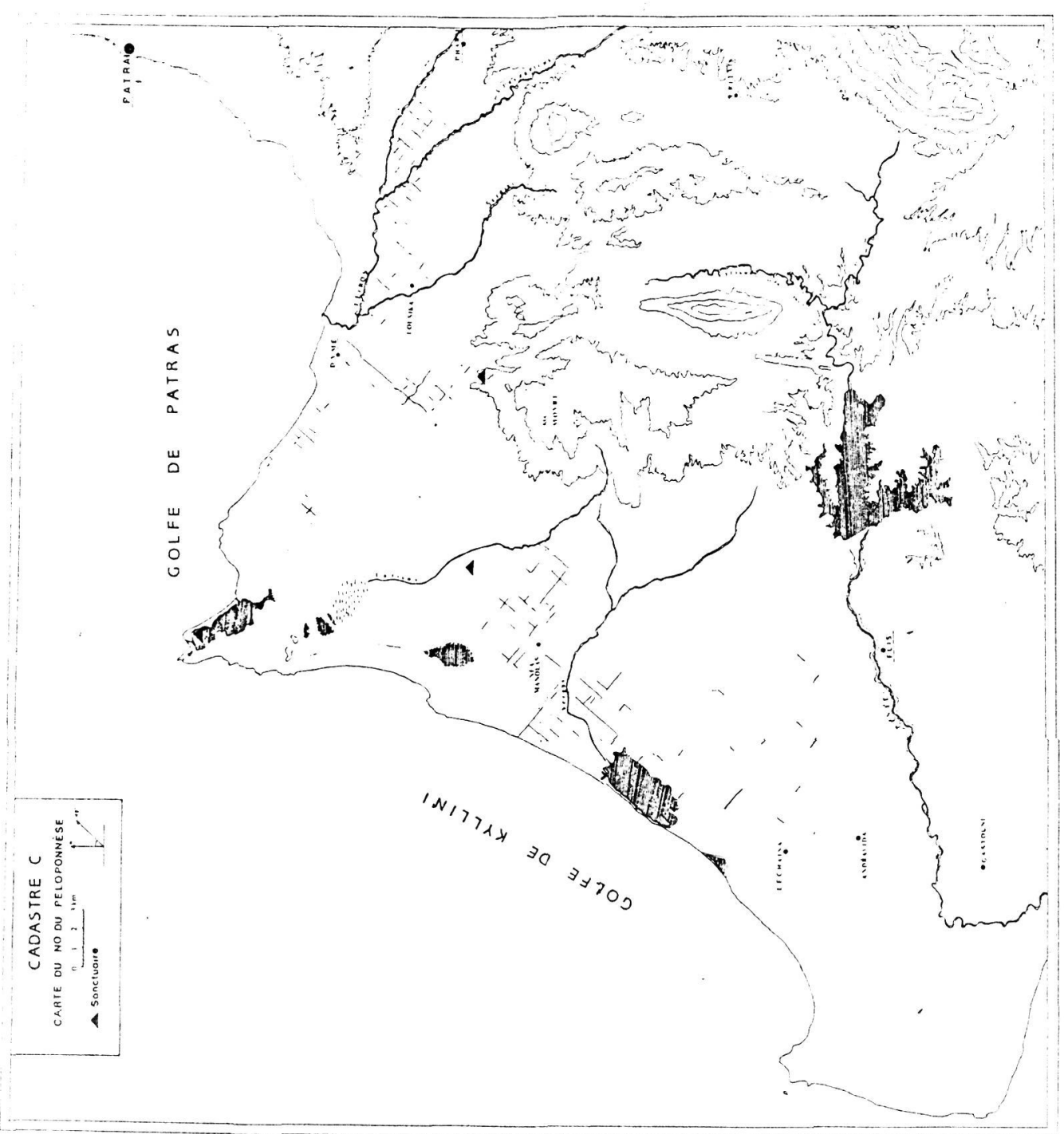


centuriation suit celle des grandes vallées du Peiros et de ses affluents qui constituent les grands axes de la communication naturelle en direction du N, NO 46 . Le relief, dans ce secteur, est très accidenté et la présence de larges et profondes vallées est un grand obstacle pour la communication NS ; celle-ci est plus facile dans le sens des vallées ce qui explique la pérennisation de ces voies, malgré l'abandon de son habitat durant de longues périodes ; ainsi il ne reste pas de traces de voies verticales qui devaient être abandonnées depuis l'antiquité. Les traces les plus denses, au sud de la vallée du Larissos, ont été repérées dans la région de Néa Manolas et de la basse vallée du fleuve Vergas, alors que vers le sud (région de Lechaina) les traces sont moins denses.

Nous avons constaté, ci-dessus que, alors que les cadastres A, B et $C$ s'étendent sur de vastes superficies fertiles de la région, certaines autres échappent à ces structures. Le premier et le second laissent de côté la fertile zone des vallées et des plateaux à l'Est ainsi que les collines et les buttes de terre qui longent la côte au Nord, les alentours immédiats d'Araxos et les piémonts du Mont Movri au Sud et au SO. Le cadastre $\mathrm{C}$ occupe surtout des zones laissées vides par les précédents, vers l'est et le sud-ouest, mais laisse aussi en dehors de la centuriation de très vastes régions : les hautes vallées du Peiros, du Parapeiros et du Serdini vers l'Est et le secteur vers la bordure montagneuse au SO au-delà du cours du Larissos. Dans les zones communes, les centuriations sont superposées 47 .

Il est intéressant de noter, ici, que les cadastres A et B sont fondés, comme nous venons de le voir, sur des axes préexistants à la colonisation; il s'agit d'une pratique qui est bien connue; l'assise d'un cadastre sur une voie préexistante n'est qu'un des cas de figures possibles pour déterminer le tracé des limites; Hygin présente l'exemple de cadastres établis à partir d'une via consularis, comme celui de Terracina sur la via Appia. Les liens de la voie antique avec

46. Sur l'orientation des cadastres romains, en fonction du relief, $\mathrm{du}$ tracé d'un fleuve ou de la côte voir J. LE GALL, Les Romains et l'orientation solaire, MEFRA 1975. 1, 287-320, particulièrement aux p. 301 sq. Voir aussi G. CHOUQUER, Le tissu rural, dans G. CHOUQUER, M. CLAVEL-LEVÊQUE, F. FAVORY et J.-P. VALLAT, Structures agraires en Italie centroméridionale, Rome 1987, 285 sq.

47. Sur la superposition de différents cadastres voir G. CHOUQUER, M. CLAVEL-LÉEEQQUE, F. FAVORY, Cadastres, occupation du sol et paysages agraires antiques, Annales ESC, 1982, 857. 
le cadastre ne sont pas toujours les mêmes; celle-ci peut être une limite mais aussi une charnière entre deux zones différentes de cadastre (ex. Rimini-Cesena et via Popilia) ou encore une simple limite de deux zones similaires 48 . Cette voie qui traversait le territoire de Dymé et d'Elis, avant d'aboutir à cette dernière ville, est bien connue dans la littérature antique et les itineraria 49 . L'orientation de ces centuriations se définit par rapport à la bordure montagneuse au sud, sud-est; il n'existe aucun rapport avec la côte 50 . Une autre caractéristique des deux premières centuriations est qu'elles n'occupent qu'une partie de la chora dyméenne en se limitant plus ou moins sur la partie plane occidentale, laissant de côté les plateaux vers l'est 51 .

La fertilité des terres, occupées par les centuriations, n'est pas la même dans tous les secteurs; elle est, certainement, plus grande vers le $S$ et le $S E$ des axes où nous avons effectivement constaté une très grande densité de signes géométriques qui correspondent aux deux centuriations. Une grande partie de l'espace cadastré, au NO des voies, semble connaître son premier aménagement avec la colonisation romaine; il n'est pas improbable que les Romains aient entrepris, à l'occasion, un système de drainage dans ce secteur marécageux où les terres argileuses sont lourdes et imperméables 52 ; je suppose que, malgré l'arpentage géométrique de la plaine, certaines terres n'ont jamais pu se mettre en culture; le petit nombre de signes conservés sur le sol en association avec celui d'installations

48. Cf. J. LE GALL (cit.p. 273 n. 46), 301 et G. CHOUQUER et F. FAVORY, Contribution a la recherche des cadastres antiques, Besançon 1979, 56-58.

49. R. BALlADIÉ, Strabon et le Péloponnèse, Paris 1980, 268-274 (présentation et commentaire des sources).

50. Cf. ci-dessus p. 273 n. 46.

51. Sur l'étendue du territoire de Dymé voir M. LACAKIS et A. RIZAKIS (éds), Cités et campagnes en Achaie: le bassin du Peiros et la plaine occidentale (sous presse).

52. En décembre de l'année 1988, pendant une période de pluies torrentielles, une grande partie de cette plaine, au SO du village de Niphoreika, était transformée en un immense lac; les travaux agricoles furent complètement suspendus; les plus tenaces des paysans prenaient de grands risques : un tracteur était enfoncé dans la terre et la marche dans ces terrains argileux, là ou il n'y avait pas d'eau, était impossible. 
humaines montrent qu'elle n'a pas connu une exploitation intensive pendant la période romaine 53 .

Les vestiges archéologiques repérés ici, indiquent que la région fut, apparemment, abandonnée à la fin de celle-ci et qu'elle est restée inoccupée jusqu'au XVe s., lorsque s'y sont établies quelques populations d'origine albanaise 54 . Quand les premiers voyageurs visitèrent le pays, il n'y avait que des forêts et des marécages et quelques hameaux et cabanes de bergers étaient situés, de même que les villages antiques, sur la frange des collines au Nord et au SE de la plaine 55 .

L'image actuelle de cette plaine ne doit pas nous tromper ; aujourd'hui, grâce aux travaux d'aménagement, de forages et de mécanisation des cultures, la région compte parmi les plus riches pour la production de pommes de terres, de maïs et de pastèques mais, pendant l'antiquité, les terres qui se trouvaient à l'ouest et au nord-ouest de la plaine étaient parmi les plus pauvres. Il est très difficile de savoir l'emplacement exact des lots primitifs des colons ; l'étendue des deux premières centuriations montre qu'ils devaient se situer soit sur la plaine occidentale soit sur les plateaux et les mottes à l'ouest du Serdini. Dans ces secteurs il y avait, naturellement, de bonnes et de mauvaises terres; il est possible, si les colons ont pris les plus vastes terres, que celles-ci n'aient pas toujours été les meilleures 56 . Ceci explique, peut-être, les échecs des colonisations successives de Dymé.

53. Pour la disposition de l'habitat dans cette zone voir ci-dessus p. 268 note 43.

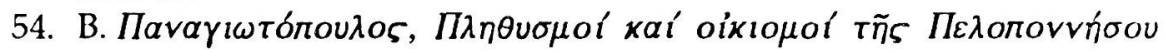

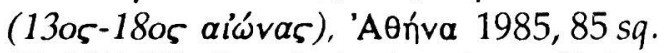

55. Cf. LEAKE, Travels in the Morea II, London 1830, 159 ; cette forêt s'étendait depuis le plateau de Kato Achaia jusqu'à Araxos à l'ouest ; on trouvera aussi une description de la plaine occidentale chez E. CURTIUS, Peloponnesos, Eine historische-geographische Beschreibung der Halbinsel, I, Gotha, 1851-1852, 423. Sur la fertilité de ces terres voir note suivante. Enfin sur la richesse, en poissons, des lagunes à l'ouest vers Araxos voir de même LEAKE, 160 et CURTIUS, 426.

56. Les axes des centuriations $\mathrm{A}$ et $\mathrm{B}$ pourraient être considérés comme la limite pour la différenciation de la qualité des terres; celles-ci n'étaient pas bonnes au nord et nord-ouest des axes car elles étaient basses alors qu'elles l'étaient au sud et sud-est des mêmes axes où le niveau du sol était plus élevé; dans cette dernière zone LEAKE (Travels, 159) remarquait que le sol est fertile mais qu'à son époque il 
Cette constatation nous oblige à nuancer l'image brutale de la colonisation romaine, confisquant aux premiers occupants pour redistribuer aux colons les meilleures terres 57 ; dans beaucoup de cas, il faut voir dans la centuriation l'instrument d'un élargissement sensible de la superficie mise en culture. A Dymé il y avait des terres disponibles qui restaient incultes faute de main d'oeuvre. Les adjectifs qu'utilisent Strabon et Plutarque 58, pour montrer le déclin démographique au Ier siècle av. J.-C., sont très évocateurs de la situation ; celle-ci fut, naturellement, améliorée par l'installation des pirates en 67 av. J.-C. ; leur expropriation forcée, trente ans plus tard, en 44 av. J.-C., par César, fournit les terres nécessaires pour ses colons. Ces deux centuriations ont laissé de côté, comme nous l'avons déjà vu, la zone des plateaux, vers l'est, et la région vallonnée, au nord de la plaine occidentale; il y avait encore, donc, des terres disponibles qui furent occupées, lors de la troisième centuriation.

La grande extension de cette dernière montre qu'elle a une toute autre fonction; car en dehors des plateaux formés par le Peiros à l'est, elle se développe sur l'ensemble de la plaine alluviale du Larissos et des autres fleuves de l'Elide, au SO; cette région faisait partie du territoire de la cité d'Elis 59 . On constate, donc, que la zone

n'était pas cultivé. Sur la richesse de la zone des plateaux et des vallées formés par les fleuves à l'est voir LEAKE, 155 qui signale parmi les produits de cette zone le riz, le coton et le maïs.

57. Il est pratiquement impossible de préciser dans le cas de Dymé, comme ailleurs, les lots primitifs des colons ; ce qui est certain est que les pentes des montagnes sont réservées aux populations locales (c'est dans ces zones que se localisent la majorité des établissements ruraux; ces zones restent, en principe, en dehors des centuriations (voir ci-dessus n. 43); il faut admettre que ces terres vu le caractère de l'agriculture antique - n'étaient pas les plus mauvaises. Sur la qualité des terres, attribuées aux colons, voir en général, G. CHOUQUER, M. CLAVEL-LÉVÊQUE, F. FAVORY (cit. p. 273 n. 47$), 861$.

58. Strab. XIV, C 665 ; Plut., Pom. $28,7$.

59. Cette région, qui correspond au Vouprassion homérique, pays du froment et de la vigne, était arrosée par le Larissos et le Vergas au sud ; cf. R. BALLADIÉ, (cit. p. 274 n. 49), ch. III, 1, 177 et 181 . Le cours $\mathrm{du}$ Larissos constituait la frontière commune entre les territoires des deux cités voisines : cf. $R E$ s.v. Larissos ; sur Elis et l'Elide, pendant cette période, voir PHILIPPSON, RE, V, 2, 1905, 2416-2421 ; 24322433. 
cadastrée ne se limite pas seulement aux terres coloniales et tributaires car elle dépasse, et de loin, la pertica de la colonie et trahit un tout autre esprit. Il ne s'agit plus, ici, d'un effort d'élargissement de l'espace cultivé qui serait attribué aux colons (cadastres $A$ et $B$ ), mais d'un aménagement qui agit indépendamment des limites juridiques et se développe en fonction d'un espace géographique et non en fonction d'une cité (ex. Italie, France: Orange); ce nouvel aménagement répond au souci de l'Empire d'organiser, dans de nouveaux cadres géométriques et rationnels, de vastes espaces. Aux exemples connus de Béziers, Valence, Rimini et des cités campaniennes peut s'ajouter celui de Dymé en Achaïe 60.

Nous avons constaté plus haut qu'il existe deux catégories de vides dans les centuriations de la chora dyméenne; la première correspond à des zones lagunaires ou marécageuses vers l'Ouest alors que la seconde comprend des secteurs de collines, de plateaux ou de piémonts. Si l'absence de traces de ceníuriation dans les premières va de soi, celle des autres nécessite une explication. Apparemment ces dernières ne pouvaient pas être centuriées, soit faute de dimension suffisante, soit pour d'autres raisons; dans tous les cas, il serait intéressant de savoir à quelle catégorie appartiennent les terres non quadrillées qui sont exceptées de la division et de l'assignation et que les agrimensores désignent selon les expressions suivantes:ager exceptus, ager extra clusus et non adsignatus ou ager subsecivus. La prospection dans ces régions a révélé, à l'exception de la grande plaine, une présence très dense d'établissements et de sanctuaires ruraux qui datent de bien avant l'arrivée des Romains dans le Péloponnèse 61 . Les terres, non quadrillées dans ce secteur, devaient appartenir aux propriétés de ces sanctuaires que les Romains, naturellement, n'avaient pas voulu confisquer, ni centurier; il peut s'agir aussi des zones qui faisaient partie du territoire des communes préexistantes; il n'y avait aucune raison d'exproprier les anciens

60. Hygin (L. 204-205) atteste, en effet, que les terres tributaires provinciales ont souvent été divisées selon le système de centuriation alors qu'il fallait, d'après lui, procéder autrement. Cette situation n'est pas unique; en effet nous avons plusicurs exemples dans lesquels la pertica peut recouvrir plusieurs territoires, y compris des cités de statut différent.

61. Le premier est situé, au SO de Dymaia, sur l'îlot de Hag. Ioannis dans la lagune de Pappas, alors que le second, dans un petit vallon, au SO du village actuel de Petrochorion. Cf. M. LACAKIS (cit. p. 268 n. 43). 
propriétaires de toutes leurs possessions ; en effet, dans ces espaces, anciennement occupés, l'existence d'un équilibre, longuement acquis, des cultures et des terroirs, imposait des formes de coexistence avec les anciens habitants; de plus la région présentait d'autres terres disponibles.

Nous avons signalé, au début de cet article, la relation entre la centuriation et les voies plus anciennes; il nous reste à évoquer la relation qui existe entre les éléments de la morphologie agraire (chemins et parcelles) et ceux qui caractérisent l'habitat rural 62 de toute taille que nous avons pu repérer dans cette région par la voie archéologique. On constate d'abord que l'installation romaine n'a pas bouleversé la forme et la répartition de l'habitat. Dans la cité, les colons semblent reprendre, après les avoir réparées, des habitations de la période précédente; s'il y a quelques nouvelles constructions, on ne constate pas une explosion du parc bâti avec la colonisation. La ville ne change pas de plan et son ampleur à l'époque romaine n'atteint pas celle de la grande période hellénistique; ses rues ont une orientation différente des cadastres 63. La présence des colons n'est pas attestée épigraphiquement dans les habitats ruraux hellénistiques dont certains survivent sous l'Empire ; il est très probable que les Romains s'installèrent dans ces villages, comme ils l'ont fait dans la cité ; il n'y avait aucune raison, dans une région urbanisée depuis longtemps, de créer de nouvelles installations pour regrouper la population récente ; celle-ci pouvait, en effet, choisir de s'installer, en dehors des cadres d'habitat traditionnel de la région, dans de petites fermes isolées dont nous avons plusieurs indications archéologiques; la présence de villae rusticae est plus rare 64 .

Si la détermination du type de limitation cadastrale et de l'orientation du système ne sont pas en soi des tâches difficiles, la précision du module demande des contrôles divers et engendre des risques. En ce qui concerne l'orientation, nous pouvons constater, ici aussi, que la physionomie de l'ensemble du territoire de la cité dicte la nature et la forme de la répartition cadastrale. Nous avons constaté que les deux centuriations sont organisées sur des axes qui dictent leurs orientations et se développent parallèlement aux

62. Cf. à ce sujet R. CHEVALLIER, La centuriation et les problèmes de colonisation romaine, 76 ; G. CHOUQUER et F. FAVORY (cit. p. 274 n. 48), 73 avec la bibliographie précédente.

63. Cf. M. LACAKIS et A. RIZAKIS, cidessus p. 274 n. 51 .

64. Cf. M. LACAKIS, ci-dessus p. 268 n. 43. 
piémonts des montagnes du Sud. Aucun de ces cadastres ne tient compte de la ligne du rivage parce qu'ils sont tous éloignés de la zone côtière. Par contre, le cadastre $C$ suit l'orientation de la vallée du Peiros qui semble correspondre avec celle du rivage, du moins, vers le NO. L'orientation des deux centuriations $\mathrm{A}$ et $\mathrm{B}$ est respectivement de $33^{\circ}$ et de $25^{\circ}$ par rapport au nord (NNO), alors que le cadastre $\mathrm{C}$ est incliné de $42^{\circ} \mathrm{E}$ (NNE). La centurie est de $699 \mathrm{~m}$ pour le premier et de $706 \mathrm{~m}$ et $710 \mathrm{~m}$ respectivement pour les deux autres.

$\mathrm{La}$ date de ces centuriations est très difficile à préciser; considérant son petit module, le cadastre A pourrait être le plus ancien et correspondre soit à l'installation des pirates de Pompée, en 67 av. J.-C., soit à la deductio de la colonie de César, en 44 av. J.-C. Le cadastre $C$ trahit, comme nous l'avons déjà dit, une nouvelle politique d'aménagement des vastes espaces qui font partie, soit du territoire des colonies romaines, soit de celui des cités avoisinantes de statut différent. Un projet si ambitieux peut bien être attribué à Auguste, surtout après la colonisation de Patras (en 16-14 av. J.-C.) ; cette nouvelle colonie devait être au centre d'un très vaste territoire 65 dont l'exploitation et le contrôle ne pouvaient s'effectuer que par le biais d'une nouvelle organisation de l'ensemble de l'espace agraire. Voilà ce qui explique la nécessité du nouveau découpage.

La pérennisation cadastrale sur certains secteurs s'explique par la continuité de l'habitat; dans les zones non habitées ou abandonnées il ne reste plus, ou peu, de traces d'une centuriation antique dans le paysage moderne. La pérennisation des formes anciennes, dans le premier cas, montre la fidélité des communautés rurales à une structure agraire adaptée à l'environnement climatique, pédologique, topographique et hydrologique du finage. Là où s'effectua une redistribution de l'habitat dans les siècles qui suivirent la centuriation, on constate des modifications de structures agraires antiques. Dans la plaine occidentale par exemple la nécessité d'une circulation NE-SO a conduit à la conservation des decumani alors que les cardines ont disparu complètement ou en partie. De même, dans la zone intérieure (vallée du Peiros) la morphologie héritée de l'ancienne centuriation et la conservation des decumani prouvent la primauté des communications E-O. Les

65. Sur la colonisation de Patras voir E. MEYER, RE XVIII, 2, 1949, col. 2210-2213; sur l'étendue de son territoire voir ibid. 2211 et U. KAHRSTEDT, Die Territorien von Patrai und Nicopolis in der Kaiserzeit, Historia I, 1950, 549-561. 
mêmes nécessités de communications justifient le maintien des cardines dans la zone des plateaux de Kato et Ano Achaia. Le cadastre romain, ici comme ailleurs, a subi, à travers les siècles, les effets des interventions humaines qui conduisirent soit à sa disparition complète soit à des déformations de la majorité de ses éléments linéaires. Malgré tout, il garde des traces tangibles d'une période d'interventions sur le paysage, liées à la transformation de Dymé en colonie romaine et à l'installation massive de colons.

(Athènes, Août 1989)

A. RIZAKIS 



$$
-1
$$

